\section{ORIGINAL PAPER}

doi: 10.5455/medarh.2016.70.351-353

Med Arch. 2016 Oct; 70(5): 351-353

Received: AUG 23, 2016 | Accepted: SEPT 29, 2016

(c) 2016 Ali Torkaman, Hamidreza Yazdi, Mohammad Ghorban Hosseini

This is an Open Access article distributed under the terms of the Creative Commons Attribution Non-Commercial License (http://creativecommons.org/licenses/ by-nc/4.0/) which permits unrestricted non-commercial use, distribution, and reproduction in any medium, provided the original work is properly cited.

\title{
The Results of Single Bundle Versus Double Bundle ACL Reconstruction Surgery, a Retrospective Study and Review of Literature
}

\author{
Ali Torkaman', Hamidreza Yazdi', Mohammad Ghorban \\ Hosseini $^{2}$ \\ 'Department of Knee Surgery, Firoozgar Hospital, Iran University of Medical Science, \\ Tehran, Iran \\ ${ }^{2}$ BIMDC Hospital, Harvard Medical School, Cambridge, USA
}

Corresponding author: Associated Professor Hamidreza Yazdi, Department of knee surgery, Firoozgar Hospital, Iran University of Medical Science, Tehran, Iran. Tel : 00989123471305. E-mail: Dr.yazdi88@yahoo.com

\begin{abstract}
Objective: The purpose of this study was to evaluate the results of single bundle and double bundle surgical techniques for anterior cruciate ligament $(A C L)$ reconstruction. Methods: In this study, all single bundle and double bundle $A C L$ reconstruction surgeries that were done in our university hospital from January 2008 to December 2012 were enrolled. All patients were followed at 2,6,12,24 weeks and 1 a 2 years post operatively. On last follow up all patients were evaluated by clinical examination, KT-1000 and Lysholm questionnaire. Results: Seventy five patients were operated using single bundle and eighty five patients with double bundle technique. Fifty seven percent of patients in single bundle and $80 \%$ of patients in double bundle group had experienced pain during follow-up period. None of cases had knee extension or flexion loss. The average side to side differences using KT1000 was $3.5 \pm 0.38$ (2.9-4.1) millimeters in single bundle group and $3.39 \pm 0.39$ (2.8-4) millimeters in double bundle group. These results showed no significant difference between two groups $(P=0.31)$. Lysholm score improved significantly in both groups, but there was no significant difference between them. Conclusion: According to this study the clinical results of single bundle $\mathrm{ACL}$ reconstruction was similar to double bundle reconstruction in short term follow up. Further studies are needed to evaluate the long term results.
\end{abstract}

Key words: ACL reconstruction, Single bundle, Double bundle, Lysholm, Patient.

\section{INTRODUCTION}

Lesions of the anterior cruciate ligament (ACL) are common orthopedic injuries. An estimated 100,000 ACL reconstructions are performed yearly in US (1). ACL reconstruction had been recommended for active patients to avoid instability and subsequent meniscus and cartilage tears (1). Investigators in recent studies reported that $63 \%-75 \%$ of patients returned to pre-injury sports activity and only $44 \%$ of patients were able to perform competitive sports activity (2). It is now widely accepted that ACL is consisted of two bundles, Anteromedial bundle (AM) that is more isometric and is responsible for anterior posterior stability spe- cially in more than 45 degrees of knee flexion and Posterolateral bundle $(\mathrm{PL})$ which is taut when knee is near extension and more responsible for rotational stability. In standard ACL reconstruction procedure AM bundle is reconstructed (3).

ACL reconstruction generally has good results, but still there are some patients who have complaints of knee pain and laxity after this procedure which demands further investigation. Some previous studies reported that non-anatomical single-bundle ACL reconstruction might cause degenerative arthritic changes because normal knee kinematics was not recovered (4-6). Recent studies had been suggested 
some techniques for ACL reconstructions focus on the restoration of anatomic double-bundle with the goal of better replicating the anatomy of the native $\operatorname{ACL}(7,8)$. Some studies have reported similar or superior results of double-bundle ACL reconstruction compared to those of single-bundle ACL reconstruction. These strategies are also supported by evidence showing that superior clinical outcomes occur when graft placement is aligned with the native $\operatorname{ACL}(9,10)$. The aim of this study was to evaluate the results of single bundle and double bundle surgical techniques for ACL reconstruction.

\section{MATERIAL AND METHODS Patients}

In this study, all patients with ACL injuries who were referred to our university hospital from January 2008 to December 2012 were enrolled. Inclusion criteria included the presence of ACL injury and the age between 18 to 45 years old and body Mass Index (BMI) less than 28 . The exclusion criteria were concomitant posterior cruciate ligament, lateral collateral ligament or medial collateral ligament injury, previous history of knee surgery or previous knee fracture and concomitant meniscal repair. ACL injuries was diagnosed by physical examination and MRI imaging as a gold standard for diagnosis confirmation (11). The study was approved by ethical research committee of Iran University of medical sciences and all of participants gave written informed consent for inclusion in the study.

\section{Surgical Technique}

All surgeries were done by two senior surgeons with the same equipment and surgical techniques. Preoperative IV antibiotic (Cephazolin 1 gram) was administered approximately 30 minutes before the incision was made. Surgery was done on supine position. General or spinal anesthesia was administered by anesthesiologist. Tourniquet was used for all patients. First arthroscopic examination was performed using two standard high anterolateral and low anteromedial portals. During arthroscopic examination, probing of ACL was done to evaluate the ligament proving a tear. Four-stranded semitendinosus and gracilis autologous grafts were used in both groups.

In single bundle group the femoral tunnel was done between AM and PL bundle foot prints using anteromedial portal. In double bundle group two anatomic tunnel on femur and two anatomic tunnel on tibia was done using AM and PL foot prints and through anteromedial portal. In every case, a button (Flipptack, Karl Storz, Tuttlingen, Germany) was used to fix each graft on femoral side, and a bioabsorbable screw (Megafix screw, Karl Storz, Tuttlingen, Germany) was used to fix each graft on tibial side. Any meniscal or chondral lesion was addressed before ACL reconstruction. Partial meniscectomy was done if necessary.

\section{Postoperative treatment and evaluation}

Postoperatively, the knee was kept in a brace in full extensions. Early ROM exercise and quadriceps muscle strengthening was encouraged in both groups. The rehabilitation program was the same in both groups. All patients were followed at 2,6,12, 24 weeks and 1 a 2 years post operatively. On last follow up all patients were evaluated by clinical examination, KT-1000 and Lysholm questionnaire

Statistical analysis

The Independent Student t-test and Chi square test were used for statistical analysis. All data were analyzed using SPSS 16.0 software. P-values less than 0.05 were considered to be significant.

\section{RESULTS}

One hundred and seventy patients were enrolled in this study. Ten patients (six patients in single bundle group and four patients in double bundle group) were excluded from the study due to loss of follow up. Seventy five patients were operated using single bundle and 85 patients with double bundle technique. ACL tear was caused by sports in $72.4 \%$, job trauma in $21.4 \%$ and accident $\mathrm{s}$ in $3.42 \%$. Fifty seven percent of patients in single bundle and $80 \%$ of patients in double bundle group had experienced pain during follow-up period. None of case had knee extension or flexion loss. The average side to side differences using KT-1000 was $3.5 \pm 0.38$ (2.9-4.1) millimeters in single bundle group and $3.39 \pm 0.39$ (2.84) millimeters in double bundle group. These results showed no significant difference between two groups $(\mathrm{P}=0.31)$.

Lysholm questionnaire result was excellent in $20.7 \%$ of patients in single bundle group and none of double bundle group. Good to excellent results had been reported in $64.3 \%$ of patients in single bundle and $80 \%$ of patients in double bundle group. Fair to good results was seen in $21.4 \%$ of patients in single bundle and $20 \%$ of patients in double bundle groups. Lysholm score improved significantly in both groups, but there was no significant difference them. Tear in medial and lateral meniscuses were present in $48.3 \%$ and $17.2 \%$ of patients respectively. Both meniscal tear was seen just in one patients. Lachman test was negative in all patients. The anterior drawer test showed firm end point in all cases.The pivot shift test was negative in all cases. No significant complication was seen in both groups.

\section{DISCUSSION}

In this study after two years follow-up, there were no significant differences between two groups based on pivot shift test, Lachman test, anterior drawer test and average side to side differences using KT-100 . The Lysholm scor was the same in both groups.

Zhang et al evaluated the results of single bundle versus double bundle ACL reconstruction. Although, all patients were satisfied and had acceptable anterior stability, the rotational stability, as evaluated by pivot shift test, was significantly superior in the double bundle technique (12). Koken et al in their study compared clinical examination, KT-1000 arthrometery, Tegner knee score, modified Cincinnati score, Knee Injury and Osteoarthritis Outcome Scale (KOOS) and International Knee Documentation Committee (IKDC) score between patients who undergone ACL reconstruction 
with single bundle or double bundle techniques. He reported that all these criteria improved after surgery in both groups and there was not a statistically difference between these two groups (13). Xue et al performed one systematic review and meta-analysis at 2012. They evaluated 17 RCTs with 1,381 patients. They found out that arthroscopic double-bundle reconstruction was associated with a lower risk of graft failures and a lower rate of positive pivot-shift test, had a lower KT1000 arthrometery measurement, a lower knee extension deficit and a higher subjective IKDC score. There was no significant difference between single-bundle and double-bundle reconstruction in Lysholm score, knee extensor peak torques, knee flexor peak torques, knee flexion deficit and objective IKDC score. They concluded that arthroscopic double-bundle reconstruction should be considered as the primary treatment in ACL reconstruction (14). In another meta-analysis done by Neel Desai, and colleagues at 2014, eight randomized controlled trials and seven prospective cohort studies and totally 970 patients were assessed. Anatomic ACL double-bundle reconstruction demonstrated less anterior laxity using KT-1000 arthrometery and less A-P laxity measured with navigation ,but did not lead to significant improvements in pivot-shift test, Lachman test, anterior drawer test, total IRER or graft failure rates compared to anatomic single-bundle ACL reconstruction. They stated that anatomic double-bundle ACL reconstruction is superior to anatomic single-bundle reconstruction in terms of restoration of knee kinematics and primarily A-P laxity. Whether these improvements of laxity result in long-term improvement of clinical outcomes remains uncertain (15). In another randomized clinical trial, Ran Sun and colleagues compared double bundle ACL reconstruction using either allograft or auto graft with single bundle ACL reconstruction. Based on the IKDC score and Lysholm score, there were no significant difference between these three groups, but they showed better anterior and rotational stability with a lower rate of arthritic progression and tunnel expansion in double bundle ACL reconstruction than single bundle procedure (16).

As mentioned, some studies are in favor of double bundle ACL reconstruction, and some demonstrated that there is no difference between these two techniques and even some showed that double bundle technique may have less appealing consequences. Those in favor of double bundle reconstruction argue that by reconstructing two bundles as anatomically as possible the results will be better than other techniques. We also try to insert the grafts as anatomically as possible but as mentioned above our result were not significantly different in single bundle versus double bundle reconstruction.

\section{CONCLUSION}

According to this study the clinical results of single bundle ACL reconstruction was similar to double bundle recostruction in short term follow up. Further studies are needed to evaluate the long term results.

Conflict of interest: none declared.

\section{REFERENCES}

1. Weninger P, Wepner F, Kissler F, Enenkel M, Wurnig C. Anatomic Double-Bundle Reinsertion After Acute Proximal Anterior Cruciate Ligament Injury Using Knotless PushLock Anchors. Arthroscopy techniques. 2015; 4(1): e1-6.

2. Ardern CL, Webster KE, Taylor NF, Feller JA. Return to sport following anterior cruciate ligament reconstruction surgery: a systematic review and meta-analysis of the state of play. British journal of sports medicine. 2011; 45(7): 596-606.

3. Markatos K, Kaseta MK, Lallos SN, Korres DS, Efstathopoulos N. The anatomy of the ACL and its importance in ACL reconstruction. European journal of orthopaedic surgery \& traumatology : orthopedie traumatologie. 2013; 23(7): 747-52.

4. Pombo MW, Shen W, Fu FH. Anatomic double-bundle anterior cruciate ligament reconstruction: where are we today? Arthroscopy: the journal of arthroscopic \& related surgery : official publication of the Arthroscopy Association of North America and the International Arthroscopy Association. 2008; 24(10): 1168-77.

5. Fithian DC, Paxton EW, Stone ML, Luetzow WF, Csintalan RP, Phelan D, et al. Prospective trial of a treatment algorithm for the management of the anterior cruciate ligament-injured knee. The American journal of sports medicine. 2005; 33(3): 335-46.

6. Ristanis S, Stergiou N, Siarava E, Ntoulia A, Mitsionis G, Georgoulis AD. Effect of femoral tunnel placement for reconstruction of the anterior cruciate ligament on tibial rotation. The Journal of bone and joint surgery American volume. 2009; 91(9): 2151-8.

7. Aglietti P, Giron F, Cuomo P, Losco M, Mondanelli N. Single-and double-incision double-bundle ACL reconstruction. Clinical orthopaedics and related research. 2007; 454: 108-13.

8. Forsythe B, Kopf S, Wong AK, Martins CA, Anderst W, Tashman $S$, et al. The location of femoral and tibial tunnels in anatomic double-bundle anterior cruciate ligament reconstruction analyzed by three-dimensional computed tomography models. The Journal of bone and joint surgery American volume. 2010; 92(6): 1418-26.

9. Yasuda K, Kondo E, Ichiyama H, Tanabe Y, Tohyama H. Clinical evaluation of anatomic double-bundle anterior cruciate ligament reconstruction procedure using hamstring tendon grafts: comparisons among 3 different procedures. Arthroscopy: the journal of arthroscopic \& related surgery: official publication of the Arthroscopy Association of North America and the International Arthroscopy Association. 2006; 22(3): 240-51.

10. Sim JA, Lee YS, Kim KO, Kim JK, Lee BK. Anatomic Double-Bundle Anterior Cruciate Ligament Reconstruction Using an Outside-in Technique: Two- to Six-Year Clinical and Radiological Follow-up. Knee surgery \& related research. 2015; 27(1): 34-42.

11. Smith TO, Lewis M, Song F, Toms AP, Donell ST, Hing CB. The diagnostic accuracy of anterior cruciate ligament rupture using magnetic resonance imaging: a meta-analysis. European Journal of Orthopaedic Surgery \& Traumatology. 2012; 22(4): 315-26.

12. Zhang Z, Gu B, Zhu W, Zhu L. Double-bundle versus single-bundle anterior cruciate ligament reconstructions: a prospective, randomized study with 2-year follow-up. European journal of orthopaedic surgery \& traumatology: orthopedie traumatologie. 2014; 24(4): 559-65.

13. Gobbi A, Mahajan V, Karnatzikos G, Nakamura N. Single-versus double-bundle ACL reconstruction: is there any difference in stability and function at 3 -year followup? Clinical orthopaedics and related research. 2012; 470(3): 824-34.

14. Li X, Xu CP, Song JQ, Jiang N, Yu B. Single-bundle versus double-bundle anterior cruciate ligament reconstruction: an up-todate meta-analysis. International orthopaedics. 2013; 37(2): 21326.

15. Desai N, Bjornsson H, Musahl V, Bhandari M, Petzold M, Fu FH, et al. Anatomic single-versus double-bundle ACL reconstruction: a meta-analysis. Knee surgery, sports traumatology, arthroscopy : official journal of the ESSKA. 2014; 22(5): 1009-23.

16. Sun R, Chen BC, Wang F, Wang XF, Chen JQ. Prospective randomized comparison of knee stability and joint degeneration for double- and single-bundle ACL reconstruction. Knee surgery, sports traumatology, arthroscopy: official journal of the ESSKA. 2015; 23(4): 1171-8. 